LA W REN CE LIVERMORE N A TIONAL LABORATORY

\title{
Tailoring properties of carbon-nanotube-based foams by ion bombardment
}

S. Charnvanichborikarn, S. J. Shin, M. A. Worsley, S. O. Kucheyev

May 24, 2012

Applied Physics Letters 
This document was prepared as an account of work sponsored by an agency of the United States government. Neither the United States government nor Lawrence Livermore National Security, LLC, nor any of their employees makes any warranty, expressed or implied, or assumes any legal liability or responsibility for the accuracy, completeness, or usefulness of any information, apparatus, product, or process disclosed, or represents that its use would not infringe privately owned rights. Reference herein to any specific commercial product, process, or service by trade name, trademark, manufacturer, or otherwise does not necessarily constitute or imply its endorsement, recommendation, or favoring by the United States government or Lawrence Livermore National Security, LLC. The views and opinions of authors expressed herein do not necessarily state or reflect those of the United States government or Lawrence Livermore National Security, LLC, and shall not be used for advertising or product endorsement purposes. 


\title{
Tailoring properties of carbon-nanotube-based foams by ion bombardment
}

\author{
S. Charnvanichborikarn, S. J. Shin, M. A. Worsley, and S. O. Kucheyev \\ Lawrence Livermore National Laboratory, Livermore, California 94550, USA
}

(Dated: May 8, 2012)

\begin{abstract}
Particle irradiation is an effective method for manipulating properties of individual carbon nanotubes (CNTs). This potential, however, remains unexplored for macroscopic assemblies of cross-linked CNTs. Here, we study structural and electrical properties of ultralow-density cross-linked CNT-based nanofoams exposed to ion radiation at room temperature over a wide range of ion masses and fluences. For all irradiation conditions studied, the electrical resistance of nanofoams initially increases with a rate that scales with the number of ballistically generated displacements. This process is attributed to the buildup of defects in graphitic nanoligaments. Irradiation with $\mathrm{Ne}$ and heavier ions leads to a decrease in the electrical resistance at large fluences, which is attributed to radiation-induced foam densification. In addition, heavy-ion bombardment causes amorphization of CNTs and smoothing of ligament surfaces. These results demonstrate that ion bombardment can be used for tailoring density, ligament morphology, and electrical properties of CNT-based foams.
\end{abstract}

Monolithic nanoporous carbons are characterized by high electrical and thermal conductivities and a tunable morphology. Such extraordinary properties make these materials promising for many energy-related applications, including electrochemical devices, ${ }^{1}$ hydrogen storage, ${ }^{2}$ catalytic supports, ${ }^{1}$ compliant electrical contacts, ${ }^{3}$ targets for inertial fusion energy, ${ }^{4}$ and energy absorbing structures. ${ }^{5}$ Conventional carbon aerogels (CAs), derived from carbonized resorcinol-formaldehyde polymeric gels, are prototypical monolithic nanoporous carbons. ${ }^{6}$ Recently, there have been a number of reports on the synthesis of carbon-nanotube (CNT) based nanofoams with improved properties compared to those of conventional CAs (see, for example, Refs. 5-11). In particular, Worsley et al. ${ }^{8}$ have developed composites of CAs with CNTs (referred to below as "CNT-CAs") with nanoligaments made of CNT bundles decorated and cross-linked by graphitic carbon nanoparticles. As a result, such CNT-CAs have unprecedented mechanical properties and low electrical resistivity. $^{8}$

Despite a larger number of previous irradiation studies of individual CNTs and variants of the non-cross-linked buckypaper (i.e., loose aggregates of individual CNTs and their bundles held together by van der Waals forces), ${ }^{12}$ the potential of ion-beam processing remains unexplored for more complex but also more technologically relevant macroscopic assemblies of cross-linked CNTs, such as CNT-CAs. Understanding radiation response of cross-linked carbon nanofoams is also crucial for their applications in a radiation environment.

Here, we investigate the effect of ion irradiation on structural and electrical properties of CNT-CAs. We find that ion bombardment results in an initial increase in the electrical resistance. This process is controlled by nuclear energy loss of ions. An order of magnitude increase in the electrical resistance is demonstrated. Further bombardment with heavy ions results in a reduction in the electrical resistance, attributed to the process of ion-beam-induced foam densification that appears to be governed by electronic energy loss of energetic ions.

The CNT-CAs studied here were synthesized as described in detail elsewhere. ${ }^{8}$ In brief, purified single-walled CNTs (Carbon Solutions, Inc.) were dispersed in water by sonication. Sol-gel precursors (resorcinol and formaldehyde) and
TABLE I: Irradiation conditions used in this study. Also given are the projected ion range $\left(R_{p}\right)$, the film thickness before irradiation $\left(h_{0}\right)$, the average (over the film thickness) nuclear $\left(\sigma_{\text {damage }}\right)$ and electronic $\left(\sigma_{\text {ionize }}\right)$ stopping powers in carbon nanoligaments, the maximum fluence $\left(\Phi_{\max }\right)$, and the initial rate of the resistance change $\left(\xi_{R}\right)$ calculated as described in the text. In all cases, bombardment was done at room temperature with the beam incident along the direction normal to the film surface with a constant beam flux of $10^{12} \mathrm{~cm}^{-2} \mathrm{~s}^{-1}$ with an energy of $3.8 \mathrm{MeV}$, except for $\mathrm{H}$ ions that were accelerated as dimers $\left(\mathrm{H}_{2}^{+}\right)$with an energy of $1.9 \mathrm{MeV} /$ atom. All fluences are given in units of atoms per area.

\begin{tabular}{ccccccc}
\hline \hline Ion & $\begin{array}{c}R_{p} \\
(\mu \mathrm{m})\end{array}$ & $\begin{array}{c}h_{0} \\
(\mu \mathrm{m})\end{array}$ & $\begin{array}{c}\sigma_{\text {damage }} \\
(\mathrm{vac} / \AA ̊ \text { /ion })\end{array}$ & $\begin{array}{c}\sigma_{\text {ionize }} \\
(\mathrm{eV} / \AA ̊ / \text { ion })\end{array}$ & $\begin{array}{c}\Phi_{\max } \\
\left(10^{16} \mathrm{~cm}^{-2}\right)\end{array}$ & $\begin{array}{c}\xi_{R} \\
\left(10^{-14} \mathrm{~cm}^{2}\right)\end{array}$ \\
\hline${ }^{1} \mathrm{H}$ & $\mathrm{x}$ & 700 & $1.1 \times 10^{-5}$ & 3.2 & 4.4 & 1.7 \\
${ }^{4} \mathrm{He}$ & $\mathrm{x}$ & 750 & $1.4 \times 10^{-4}$ & 25 & 1.9 & 11 \\
${ }^{20} \mathrm{Ne}$ & $\mathrm{x}$ & 120 & $1.5 \times 10^{-2}$ & 170 & 0.5 & 440 \\
${ }^{40} \mathrm{Ar}$ & $\mathrm{x}$ & 50 & $4.5 \times 10^{-2}$ & 210 & 0.2 & 1040 \\
${ }^{129} \mathrm{Xe}$ & $\mathrm{x}$ & 50 & $5.0 \times 10^{-1}$ & 190 & 1.2 & 4940 \\
\hline \hline
\end{tabular}

the polymerization catalyst $\left(\mathrm{NaCO}_{3}\right)$ were added, and the mixture gelled. Wet gels were washed with acetone, dried with supercritical $\mathrm{CO}_{2}$, and pyrolyzed at $1050{ }^{\circ} \mathrm{C}$ under $\mathrm{N}_{2}$. Pyrolyzed monoliths, with a density of $\sim 30 \mathrm{mg} \mathrm{cm}^{-3}$ and a CNT loading of $\sim 50 \mathrm{wt}$. $\%$, were machined with a cylindrical endmill to thicknesses much smaller than projected ion ranges (Table I) and placed on insulating substrates (either quartz or sapphire). Conductive silver paste was used to make electrical contacts.

Table I gives the details of the irradiation experiments performed. ${ }^{13}$ Ion ranges and stopping powers were calculated with the TRIM code (version SRIM-2011.08). ${ }^{14-16}$ The electrical resistance of films was measured after each fluence increment while the sample remained under vacuum. Following irradiation to the maximum fluences (also given in Table I), the samples were examined by scanning electron microscopy (SEM) in a JEOL 7401-F microscope operated at $2 \mathrm{kV}$ and by bright-field transmission electron microscopy (TEM) in a FEI 


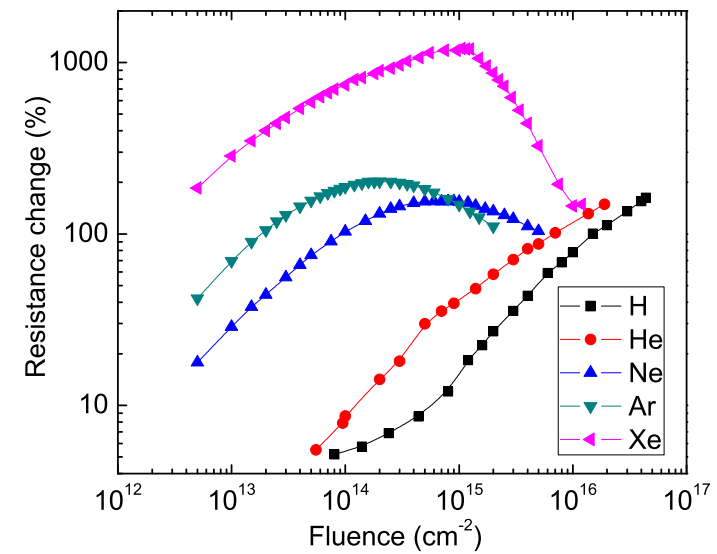

FIG. 1: (Color online) Ion fluence dependences of the change in the electrical resistance of CNT-based nanofoams. The details of irradiation conditions are given in Table I.

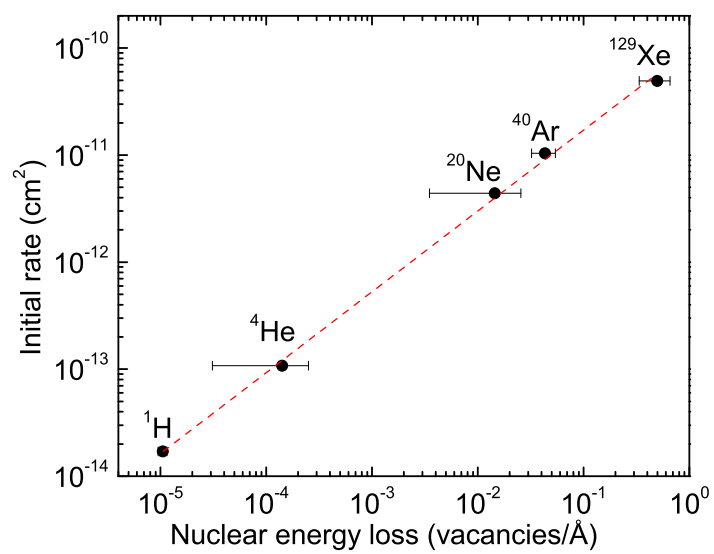

FIG. 2: (Color online) The initial rate of the change of the electrical resistance $\left(\xi_{R}\right)$ of CNT-based nanofoams as a function of the nuclear stopping power of ions in full-density carbon nanoligaments. Horizontal error bars represent the stopping power variation through the film thickness. Dash line is a fit, revealing a power law with an exponent of $0.76 \pm 0.02$.

\section{TF-20 Tecnai microscope operated at $200 \mathrm{kV}$.}

Figure 1 shows a summary of ion fluence dependences of the fractional change in the electrical resistance, defined as $\left(R-R_{0}\right) / R_{0}=\Delta R / R_{0}$, where $R_{0}$ and $R$ are sample resistances before and after irradiation, respectively. It is seen from Fig. 1 that, for all the ion species studied, irradiation initially results in a monotonic increase in $\Delta R / R_{0}$. A similar resistance behavior has been reported for full-density graphite exposed to neutron irradiation in a reactor. ${ }^{17}$ This suggests that, at the initial stage of irradiation, the electrical resistance of CNT-CAs is related to irradiation-induced changes in the electrical resis-
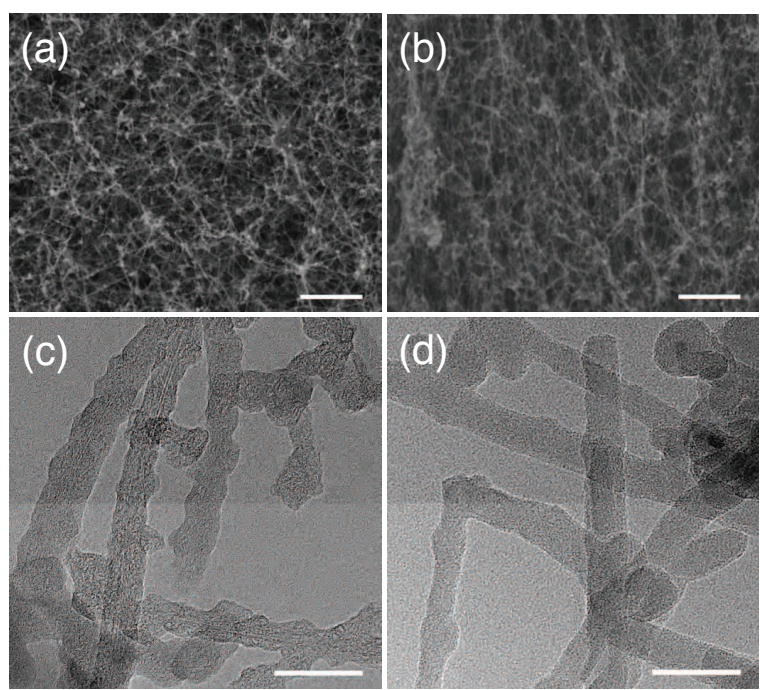

FIG. 3: (a),(b) SEM and (c),(d) TEM images of (a),(c) the assynthesized CNT-based nanofoam and (b),(d) a nanofoam irradiated with $3.8 \mathrm{MeV} \mathrm{Xe}$ ions to a fluence of $1.2 \times 10^{16} \mathrm{~cm}^{-2}$. The scale bars are $1 \mu \mathrm{m}$ and $50 \mathrm{~nm}$ for SEM and TEM images, respectively.

tivity of nanoligaments. Primak and Fuchs ${ }^{18}$ have described this behavior empirically by a hyperbola

$$
\frac{\Delta R}{R_{0}}=\frac{A \xi_{R} \Phi}{A+\xi_{R} \Phi}
$$

where $A$ is a constant reflecting the maximum $\Delta R / R_{0}$ value for large doses, $\xi_{R}$ is the initial rate of resistance change, and $\Phi$ is ion dose. Despite the fact that saturation at $A$ has not been experimentally observed in either our (Fig. 1) or previous studies, ${ }^{17-19}$ the above equation can still be use to fit fluence dependences in order to evaluate the initial rate of the resistance change $\left(\xi_{R}\right)$. Table I and Fig. 2 show the dependence of $\xi_{R}$ on the nuclear stopping power of ions in full-density carbon nanoligaments. It is clearly seen that $\xi_{R}$ is governed by the nuclear (elastic) energy loss processes. The dash line in Fig. 2 shows a linear fit in double logarithmic coordinates, demonstrating that the $\xi_{R}$ rate depends on the nuclear stopping power slightly sublinearly, following a power law with an exponent of $0.76 \pm 0.02$. The electrical resistivity of carbon nanoligaments depends on the number of ion-beam-produced defects that are expected to result in a slight increase in the concentration of free carriers and a strong decrease in the carrier mobility. ${ }^{17}$ The combined effect is a continuous increase of the electrical resistivity with increasing irradiation fluence, ${ }^{17}$ as revealed by Fig. 1 for the early stage of irradiation for all the ion species studied.

Figure 1 also shows that, for irradiation with Ne and heavier ions, the ion fluence dependence of $\Delta R / R_{0}$ exhibits a peak, and the resistance decreases at large fluences. For the maximum doses used here, the resistance is, however, not fully recovered. The fluences corresponding to $\Delta R / R_{0}$ maxima are $\sim 7 \times 10^{14} \mathrm{Ne} \mathrm{cm}^{-2}, \sim 2 \times 10^{14} \mathrm{Ar} \mathrm{cm}^{-2}$, and $\sim 1 \times 10^{15} \mathrm{Xe}$ $\mathrm{cm}^{-2}$. Such peak fluences do not scale with the electronic, 
nuclear, total (electronic and nuclear combined) energy deposited in the film (Table I).

The complex behavior of $\Delta R / R_{0}$ for heavy ions revealed by Figs. 1 and 2 can be understood by noting that the electrical resistance of nanoporous materials is determined by two fundamentally different contributions: (i) by the resistivity of nanoligaments that depends on the concentration and type of lattice defects present and (ii) by the monolith density and the geometry and connectivity of nanoligaments (i.e., the way of how the nanoligaments are interconnected into a three-dimensional macroscopic assembly). We have found that heavy-ion irradiation results in the densification of CNT-CAs, evidenced as a change in the film thickness resulting in the appearance of a surface step between irradiated and unirradiated (i.e., masked during irradiation) regions of the film. For example, the film thickness reduces from $\sim 50$ to $\sim 10 \mu \mathrm{m}$ as a result of 3.8 $\mathrm{MeV}$ Xe ion irradiation to the maximum fluence of $1.2 \times 10^{16}$ $\mathrm{cm}^{-2} \cdot{ }^{20}$ Such densification is expected to decrease the electrical resistance since the electrical resistivity of CNT-CAs depends on the monolith density super-linearly with an exponent of $\sim 1.6{ }^{8}$ In fact, the fluence dependences of $\Delta R / R_{0}$ from Fig. 1 can be described quantitatively by a simple analytical model that combines (i) the empirical hyperbolic fluence dependence of the resistivity of carbon nanoligaments (Eq. 1), (ii) foam densification (assumed to be exponential with a saturation at a density limited by the full-density of graphitic carbon), and (iii) a power law dependence of the film electrical resistance on the monolith density. ${ }^{8}$ However, such a model has very limited predictive capabilities since more work is currently needed to understand the physics behind the empirical hyperbolic law (Eq. 1) and the behavior of radiation-induced densification of CNT-CAs.

Figures 3(a) and 3(b) compare SEM images of CNT-CAs before and after irradiation, respectively, with Xe ions to the maximum fluence studied. These figures reveal that, despite a pronounced irradiation-induced densification observed at the macroscale, the morphological structure of CNT-CAs, consisting of a network of randomly interconnected large-aspectratio nanoligaments, is essentially indistinguishable for unirradiated and irradiated samples. Such negligible morphological changes have also been confirmed by SEM imaging for all the irradiation conditions studied (Table I). Hence, ion- induced foam densification appears to proceed via a decrease in the effective aspect ratio of ligaments rather than via a growth of nanoligament diameters (as is, for example, the well known case of thermally-induced densification of nanoporous glasses driven by total energy minimization).

Figures 3(c) and 3(d) compare TEM images of CNT-CAs before and after irradiation with Xe ions, respectively. Walls of CNTs are clearly visible in nanoligaments of virgin CNTCAs [Fig. 3(c)]. Bombardment with Xe ions results in lattice amorphization: no CNT walls are visible in ligaments of the Xe-ion-irradiated CNT-CA [Fig. 3(d)]. Such an amorphization is consistent with a number of previous reports of ion-induced amorphization of CNTs. ${ }^{12}$ More importantly, a comparison of TEM images such as shown in Figs. 3(c) and 3 (d) reveals that irradiation results in smoothing nanoligament surfaces. We attribute this smoothing to the process of ballistic sputtering of nanoligaments with material redeposition on surfaces of adjacent nanoligaments. Such a sputtering/redeposition process is expected to reduce the roughness of ligament surfaces since the ballistic sputtering yield scales with the area of the surface formed by the overlap of the ballistic collision cascade with the ligament surface.

In conclusion, we have demonstrated that ion bombardment can be used to tailor the electrical resistance, the monolith density, as well the crystallinity and geometry of nanoligaments of ultralow-density cross-linked CNT-based nanofoams. Results suggest that radiation-induced changes in the electrical resistance are dominated by two competing processes: (i) the defect production governed by nuclear energy loss according to a sublinear power law dependence with an exponent of $0.76 \pm 0.02$ and (ii) foam densification that is more complex and does not scale with either nuclear or electronic energy loss. More work is currently needed to better understand radiation damage processes in CNT-based macroassemblies.

This work was performed under the auspices of the U.S. DOE by LLNL under Contract DE-AC52-07NA27344. Electron microscopy experiments were conducted at the National Center for Electron Microscopy, Lawrence Berkeley National Laboratory, which is supported by the U.S. Department of Energy under Contract No. DE-AC02-05CH11231.
1 See, for example, a review by D. R. Rolison, R. W. Long, J. C. Lytle, A. E. Fischer, C. P. Rhodes, T. M. McEvoy, M. E. Bourga, A. M. Lubers, Chem. Soc. Rev. 38, 226 (2009).

2 H. Kabbour, T. F. Baumann, J. H. Satcher, Jr., A. Saulnier, and C. C. Ahn, Source, Chem. Mater. 18, 6085 (2006).

3 G. Toth, J. Malkin, N. Halonen, J. Palosaari, J. Juuti, H. Jantunen, K. Kordas, W. G. Sawyer, R. Vajtai, and P. M. Ajayan, Adv. Mater. 21, 2054 (2009).

${ }^{4}$ See, for example, a review by S. O. Kucheyev and A. V. Hamza, Appl. Phys. Rev. 108, 091101 (2010).

5 A. Cao, P. L. Dickrell, W. G. Sawyer, M. N. Ghasemi-Nejhad, and P. M. Ajayan, Science 310, 1307 (2005).

6 See, for example, a recent review by A. M. Elkhatat and S. A. Al-Muhtaseb, Adv. Mater. 23, 2887 (2011).
7 M. B. Bryning, D. E. Milkie, M. F. Islam, L. A. Hough, J. M. Kikkawa, and A. G. Yodh, Adv. Mater. 19, 661 (2007)

8 M. A. Worsley, S. O. Kucheyev, J. H. Satcher, A. V. Hamza, and T. F. Baumann, Appl. Phys. Lett. 94, 073115 (2009).

9 X. Gui, J. Wei, K. Wang, A. Cao, H. Zhu, Y. Jia, Q. Shu, and D. Wu, Adv. Mater. 22, 617 (2010).

10 M. Xu, D. N. Futaba, T. Yamada, M. Yumura, and K. Hata, Science 330, 1364 (2010).

11 P. D. Bradford, X. Wang, H. Zhao, and Y. T. Zhu, Carbon 49, 2834 (2011).

12 See, for example, a review by A. V. Krasheninnikov and K. Nordlund, J. Appl. Phys. 107, 071301 (2010).

13 Ion irradiation was carried out with the $4 \mathrm{MV}$ ion accelerator (National Electrostatics Corporation, model $4 \mathrm{UH}$ ) at Lawrence Liv- 
ermore National Laboratory.

14 J. F. Ziegler, J. P. Biersack, and U. Littmark, The Stopping and Range of Ions in Solids (Pergamon, New York, 1985), Vol. 1, p 109.

15 Note that TRIM-code calculations typically underestimate the $R_{p}$ for implantation of heavy ions into light substrates. ${ }^{16}$ Hence, actual $R_{p}$ values are likely larger than those given in Table I.

16 P. L. Grande, F. C. Zawislak, D. Fink, and M. Behar, Nucl. In- strum. Methods Phys. Res. B 61, 282 (1991).

17 See, for example, B. T. Kelly, Physics of Graphite (Applied Science Publishers, London, 1981), P. 455.

18 W. Primak and L. H. Fuchs, Phys. Rev. 103, 541 (1956).

19 M. Burton and T. J. Neubert, J. Appl. Phys. 27, 557 (1956).

20 Radiation-induced densification is also consistent with our recent (unpublished) results for irradiation of CNT-CAs with $2 \mathrm{MeV} \mathrm{Ar}$ and Xe beams. 